\title{
Cyclic Poly( $\alpha$-peptoid)s by Lithium bis(trimethylsilyl)amide (LiHMDS)-mediated Ring-Expansion Polymerization: simple access to bioactive backbones
}

\author{
Pedro Salas-Ambrosio, ${ }^{\mathrm{a}}$ Antoine Tronnet, ${ }^{\mathrm{b}, \mathrm{e}}$ Marc Since, ${ }^{\mathrm{c}}$ Sandra Bourgeade-Delmas, ${ }^{\mathrm{d}}$ Jean-Luc Stiglia- \\ ni, ${ }^{\mathrm{b}}$ Amelie Vax, ${ }^{\mathrm{a}}$ Sébastien Lecommandoux, ${ }^{\mathrm{a}}$ Bruno Dupuy, ${ }^{\mathrm{e}}$ Pierre Verhaeghe, ${ }^{* \mathrm{~b}}$ Colin Bonduelle*a \\ ${ }^{a}$ Univ. Bordeaux, CNRS, Bordeaux INP, LCPO, UMR 5629, F-33600, Pessac, France. \\ ${ }^{\mathrm{b}}$ LCC-CNRS, UPR8241, Université de Toulouse, CNRS, UPS, Toulouse, France. \\ ${ }^{\mathrm{c}}$ Normandie Univ, UNICAEN, CERMN, 14000, Caen, France. \\ d UMR 152 PHARMA-DEV, Université de Toulouse, IRD, UPS, Toulouse, France. \\ ${ }^{\mathrm{e}}$ LPBA, Institut Pasteur, UMR-CNRS 2001, Université de Paris, Paris, France.
}

\begin{abstract}
Cyclic polymers display unique physicochemical and biological properties. However, their development is often limited by their challenging preparation. In this work, we present a simple route to cyclic poly( $\alpha$-peptoids) from $N$-alkylated- $N$ carboxyanhydrides (NNCA) using LiHMDS promoted ring-expansion polymerization (REP) in DMF. This new method allows the unprecedented use of lysinelike monomers in REP to design bioactive macrocycles bearing pharmaceutical potential against Clostridioïdes difficile, a bacterium responsible for nosocomial infections.
\end{abstract}

Cyclic polymers are unique macromolecules that have different physical properties than their linear counterparts due to the "double constraint" related to the cyclization. For instance, cyclic polyesters demonstrated modified solubility, ${ }^{1,2}$ crystallinity ${ }^{2,3}$ and improved degradation profile against chemical hydrolysis. ${ }^{4}$ Theoretically, the radius of gyration of a cyclic polymer is expected to be $1 / \sqrt{ } 2$ that of the linear one in unperturbed conditions. ${ }^{5}$ With cyclic diblock copolymers, the models predict a transition from the disordered to an ordered phase in bulk at $(\chi N)_{c}=17.8$ for a symmetric diblock copolymer as compared to linear diblocks for which $(\chi N)_{c}=10.5 .^{6}$ In solution, cyclic polymers possess a ring-like topology displaying smaller hydrodynamic volume and it has been shown that cyclization concentrates spatial distribution of the side chains to achieve unique biological properties. ${ }^{7,8}$ For these reasons, efficient syntheses of cyclic polymers have attracted great interest in recent years.
So far, three main routes have been proposed to synthesize cyclic polymers. ${ }^{7,9,10}$ Two of these routes involve a post-polymerization grafting using tailored extremities of linear polymers and uni- or bimolecular couplings. ${ }^{11-13}$ In these two options, the synthesis of polymers remains difficult in marked contrast to the third option, named ring-expansion polymerization (REP), that is far more efficient. ${ }^{1,14}$ REP involves the insertion of cyclic monomers via the use of specific catalyst/initiator systems and it has been used in metathesis polymerization to produce cyclic olefins, ${ }^{15}$ or in ring-opening polymerization (ROP) to produce cyclic polyesters ${ }^{14,16}$ and polypeptides. ${ }^{17} \mathrm{~N}$-heterocyclic carbenes (NHCs) have provided spectacular results in this direction by promoting REP with evolving growth control. ${ }^{14,17,18}$

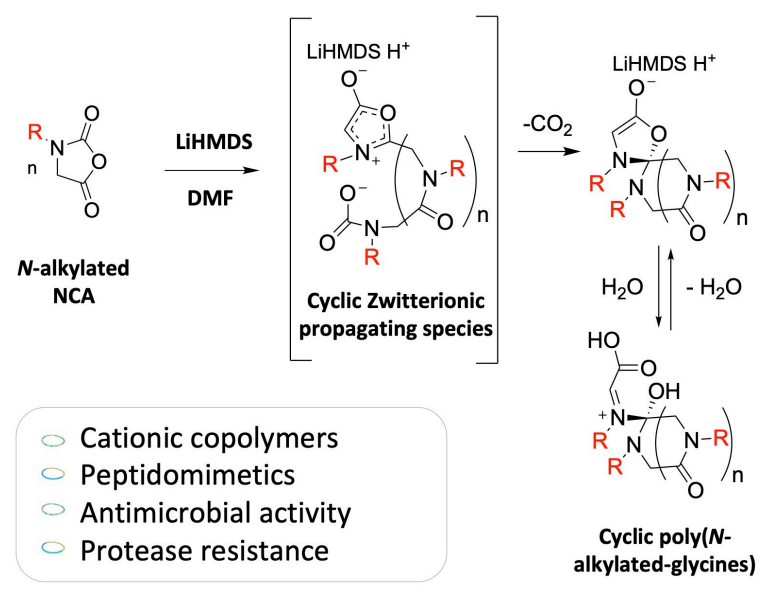

Scheme 1. LiHMDS-mediated REP of NNCA: cyclic and bioactive species are produced in a controlled fashion. 
This work is focused on the synthesis of cyclic poly $(\alpha$-peptoid)s using the ROP of glycine-based $N$ alkylated- $N$-carboxyanhydrides (NNCA). ${ }^{19}$ D. Zhang and her collaborators carried out pioneer works about such cyclic structures: they have shown that $\mathrm{NHC}^{18}$ and 1,8-diazabicycloundec-7-ene (DBU) ${ }^{20}$ can afford cyclic polypeptoids from NNCA. Although being quite efficient, these approaches remain sensitive to solvent effects, limiting the scope of the monomers that can be used for solubility reasons. ${ }^{18,21}$ Herein, we report an alternative approach leading to another REP of NNCA that can be performed in a more polar medium (DMF), using lithium bis(trimethylsilyl)amide (LiHMDS). LiHMDS is a strong base already known to promote the superfast ROP of non-alkylated NCA monomers. ${ }^{22}$ Unexpectedly, in this work, the use of LiHMDS allowed the synthesis of cyclic polypeptoids when mixed with various NNCA: sarcosine-NCA (Sar-NCA), $\mathrm{N}$-benzyl-NCA ( $\mathrm{N}$-phe-NCA) or $\mathrm{N}$ - $\mathrm{Cbz}$ aminobutyl-NCA (Z-N-lys-NCA). Using those monomers, the LiHMDS methodology allows simple access to cationic macromolecules with interesting antibacterial activities (Scheme 1).

As part of a catalytic study aiming at synthesizing polysarcosine (poly(sar)), we first implemented the ROP of Sar-NCA at 0.4 M in DMF from hexylamine, with or without a stoichiometric amount of LiHMDS (as compared to hexylamine) at a monomer/LiHMDS ratio $(\mathrm{M} / \mathrm{B}$; $\mathrm{B}$ stands for base $)=100$, leading to poly(sar) 1 and 2, (table 1). Monitoring the disappearance of the NNCA peak by FTIR (figure S3) revealed that the Sar-NCA stretching was disappearing at a faster rate of $k=9.5 \times 10^{-3} \mathrm{~min}^{-1}$ in absence of hexylamine (poly(sar) 3). Upon purification, polymer 3 was characterized by ${ }^{1} \mathrm{H}$ NMR and the SEC analysis revealed a lower elution time and a narrow dispersity (figures 1A, S4 and table 1). MALDI analyses were then carried out to clarify the structure of $\mathbf{3}$ : it revealed the presence of three sets of ions matching $\Delta \approx 71$ $\mathrm{g} / \mathrm{mol}$ with an initiation attributable to previously observed spirocyclic structures (figure 1B). ${ }^{21}$ So far, the existence of cyclic poly( $\alpha$-peptoid $) \mathrm{s}$ in solvents with high dielectric constants such as DMF was never reported. In this work, ESI-MS and FTIR spectra run during the early stages of the polymerization (figures S6-7) highlight most of the key intermediates of a possible REP mechanism starting from Sar-NCA deprotonation (scheme S4.4). ${ }^{21}$ Moreover, DFT calculations carried out on a model zwitterionic intermediate ( 5 monomer unit) show that the cyclic form is energetically more favorable than the linear one $(\Delta G=$ $-18.6 \mathrm{KJ} / \mathrm{mol}$, figure $\mathrm{S} 8$ ). As depicted in figure 2 , the chemical structure of this intermediate includes LiHMDS and involves three stabilizing interactions between the polymer and the $\mathrm{Li}^{+}$cation, allowing spatial proximity between the anionic carbamoyl extremity and the positive charge of the Münchnone moiety (5-hydroxy-2,3-dihydrooxazolium enolate $=5$ $\mathrm{OH}-\mathrm{DHE}$ in figure 2).

A)

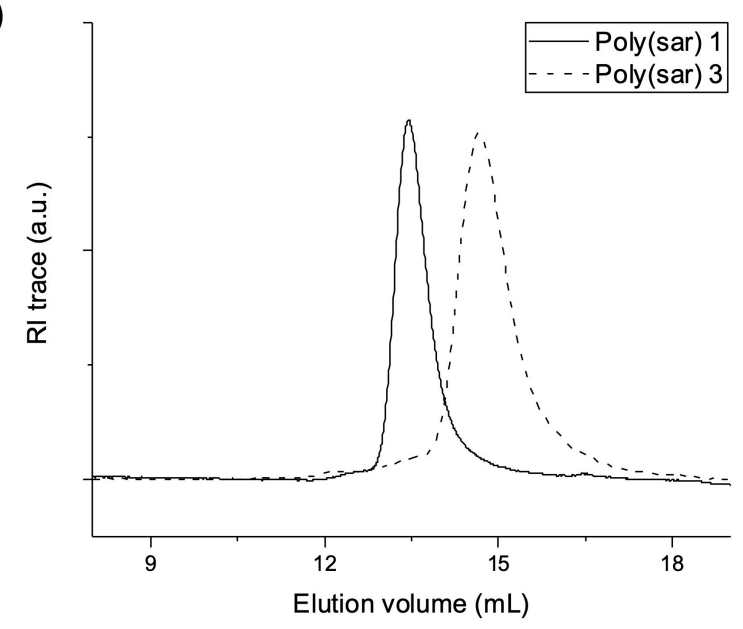

B)
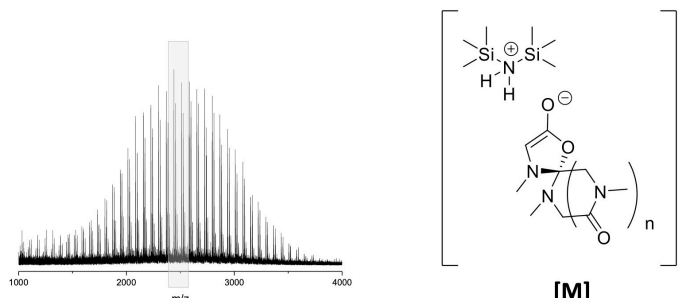

[M]

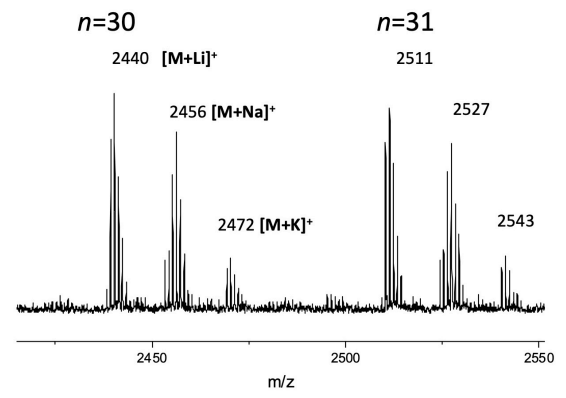

Figure 1. Macromolecular characterizations A) SEC characterization in DMF (1\% LiBr) of poly(sar) 1 and poly(sar) 3. B) Full MALDI-ToF spectra with assignment of the individual peaks from poly(sar) 1 .

By decreasing the $\mathrm{M} / \mathrm{B}$ ratio to 35 (an increase in dispersity was observed for lower ratios), polymer 4 was synthesized to confirm the presence of a Münchnone ring on the macromolecule. ${ }^{1} \mathrm{H}$ and ${ }^{13} \mathrm{C}$ NMR spectra of this new polymer corroborated the proposed Münchnone specie (figures S10 and S13) and FTIR analyses showed both the carbonyl stretching of the zwitterionic structure and the Münchnone initiation species at 1568 and $1759 \mathrm{~cm}^{-1}$ as well as the polymer backbone at $1639 \mathrm{~cm}^{-1}$ (figure S11). ${ }^{23}$ It is to note that the mesoionic oxazole moiety is known to ring-open, in acidic conditions ${ }^{24}$ (scheme S4.5). Thus, the reactivity of the 5-OH-DHE ring from the cyclic structures was first evaluated by exposing polymer 4 
to $\mathrm{HBr}$ (2 equiv.) in TFA. After this treatment, MALDI-TOF and ${ }^{13} \mathrm{C}$ NMR spectra indeed revealed possible ring-opening into an iminium acid derivative (figures S12-S14) without modification of the overall macrocyclic topology, as attested by NMR, MALDITOF and SEC analyses (figure S15). On another hand, cyclic poly $(\alpha$-peptoid) were reported to react with acyl chlorides to afford the linear counterparts. ${ }^{21}$ Accordingly, MALDI-TOF analyses revealed that the treatment of cyclic polymer 4 with propionic anhydride (2 equiv.) afforded a linear analogue of the polymer for which the SEC analysis revealed a higher molar mass (figures S15-18). Similar propylation was repeated with polymer 3 and, in agreement with previous reports, ${ }^{18}$ the more compacted structure of the cyclic polymer (before propylation) was reflected by a larger elution volume at the same molar masses (figure $3 \mathrm{~A}$ and S15).

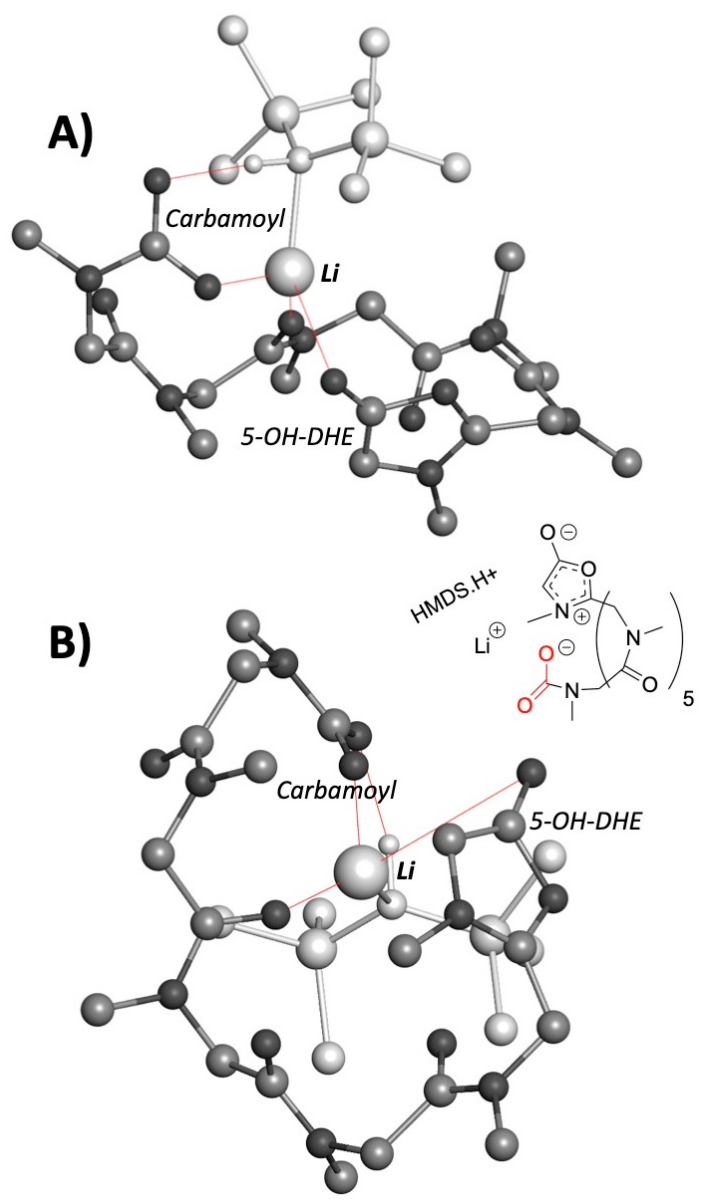

Figure 2. The most stable zwitterionic intermediate upon DFT calculation (basis M062X/6-31+G(d,p)) in DMF. A) front view and B) top view. The polymer backbone is colored black. Red lines show stabilizing interactions between $\mathrm{Li}^{+}$, the polymer chain, the carbamoyl extremity and the Münchnone moiety (5-OHDHE).
To further study the LiHMDS-induced polymerization, several REP of Sar-NCA were carried out in DMF by varying the M/B ratio from 60 to 400 (yield: $89 \%-97 \%$, table S5). SEC characterizations were performed and all polymers presented increasing $M_{w}$ values with controlled dispersities $(\mathrm{Ð}=1.03-1.08$, table 1 and figure 3B). The lower the amount of LiHMDS added, the higher the weighted-average molecular weight $\left(M_{w}\right)$, and an unexplained deviation was observed, especially for the higher ratios. For all polymers, the $M_{w}$ cyclic $/ M_{w}$ after propylation from SEC analysis were close to 0.8 , the value established with cyclic polypeptoids when formed from NHCs promoted REP $\left(<\mathrm{G}>\right.$, table 1 and ESI) ${ }^{25,26}$ Ultimately, the living nature of the REP was evaluated. Synthesis of poly(sar) at $\mathrm{M} / \mathrm{B}=50$ was first performed and, at full conversion, another feed of Sar-NCA $(\mathrm{M} / \mathrm{B}=50)$ was added to the reaction until it was also consumed (FTIR monitoring). The resulting poly(sar) 9 presented a $M_{w}$ of 3200 g. $\mathrm{mol}^{-1}$ close to the $M_{w}$ of polymer 3 and associated with a low dispersity of $\mathrm{Ð}=1.1$ (table 1). This result indicated that diblocks could be designed using LiHMDS.
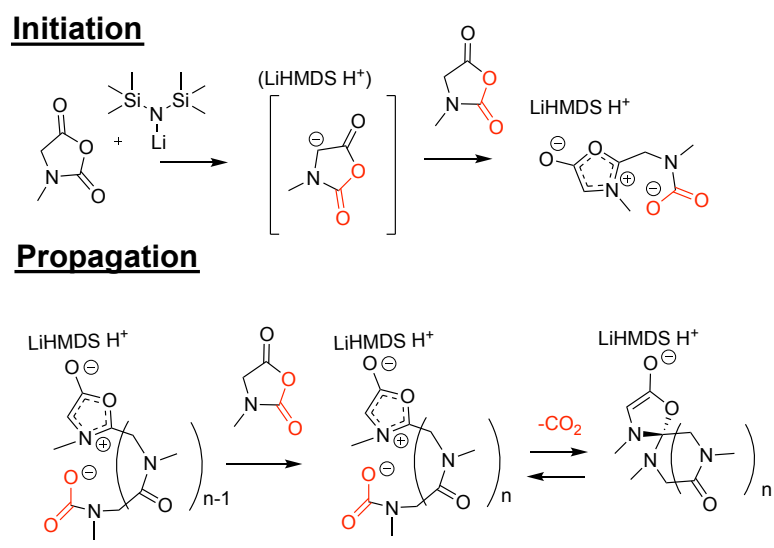

Scheme 2. Postulated basic mechanism for LiHMDS-mediated REP of NNCA.

Table 1. LiHMDS-mediated REP of Sar-NCA $(\mathrm{B}=$ LiHMDS). Mass relation $<\mathrm{G}>\left(M_{w}\right.$ cyclic $/ M_{w}$ after propylation) provides a clue regarding the radius gyration. Molar masses were determined by SEC using MALS detection in DMF ( $\mathrm{LiBr} 1 \%)$. ${ }^{\text {a Theoretical }} M_{w}$ taking $\mathrm{B}$ as initiator; ${ }^{\mathrm{b}}$ Polymerization degree $\left(\mathrm{DP}=M_{n} / 71 \mathrm{~g}\right.$ $\left.\mathrm{mol}^{-1}\right) ;{ }^{\mathrm{c}} \mathrm{B}$ stands for hexylamine.

\begin{tabular}{|c|c|c|c|c|c|c|}
\hline & M/B & $\begin{array}{c}\text { Theo. } M_{w} \\
(\mathrm{~g} / \mathrm{mol})^{\mathrm{a}}\end{array}$ & $\begin{array}{c}M_{\mathrm{w}} \\
(\mathrm{g} / \mathrm{mol})\end{array}$ & $\begin{array}{c}\text { DP from } \\
\text { SEC }^{\mathrm{b}}\end{array}$ & $\mathrm{Ð}$ & $<\mathrm{G}>$ \\
\hline Poly(sar) 1 & $100^{\mathrm{c}}$ & 7100 & 6100 & 89 & 1.06 & - \\
\hline Poly(sar) 2 & 100 & 7100 & 2300 & 33 & 1.29 & - \\
\hline Poly(sar) 3 & 100 & 7100 & 3500 & 47 & 1.06 & 0.88 \\
\hline Poly(sar) 4 & 35 & 2500 & 1800 & 24 & 1.03 & 0.81 \\
\hline Poly(sar) 5 & 60 & 4200 & 2100 & 29 & 1.04 & 0.88 \\
\hline Poly(sar) 6 & 75 & 5300 & 2600 & 34 & 1.08 & 0.89 \\
\hline Poly(sar) 7 & 200 & 14200 & 5000 & 66 & 1.06 & 0.83 \\
\hline
\end{tabular}




\begin{tabular}{|c|c|c|c|c|c|c|}
\hline Poly(sar) 8 & 400 & 28400 & 8200 & 115 & 1.04 & - \\
\hline Poly(sar) 9 & $50+50$ & 7100 & 3200 & 40 & 1.10 & - \\
\hline
\end{tabular}

A)

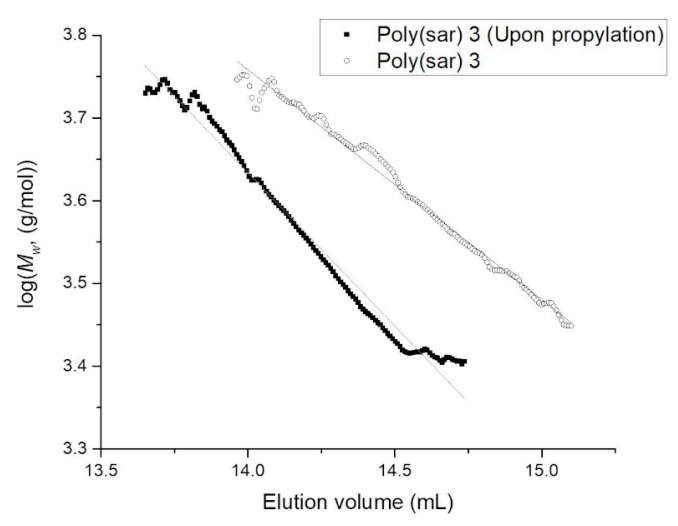

B)

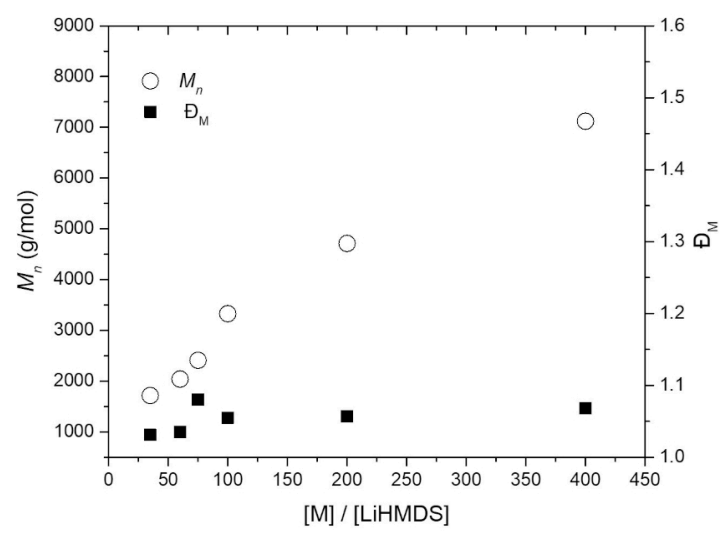

Figure 3. LiHMDS-mediated REP of Sar-NCA. A) Difference of elution profiles of polymer 3 before or after propylation versus the $\log M w(\mathrm{~g} / \mathrm{mol})$. The difference in slope is hypothetically explained by a slight contamination of 3' with cyclic structures. B) Evolution of $M_{n}$ and $\bigoplus_{\mathrm{M}}\left(\mathrm{M}_{\mathrm{w}} / \mathrm{M}_{\mathrm{n}}\right)$ with M/LiHMDS ratio (determined by SEC in DMF using a $\mathrm{dn} / \mathrm{dc}=0.0978$ ).

Cyclic structures are used by living systems to achieve specific bioactivities. ${ }^{27}$ This encompasses cyclic antimicrobial peptides (cAMP) that are cationic and broad-spectrum anti-infective backbones. ${ }^{28}$ The use of a simple REP methodology, working in DMF, opens up interesting prospects to design simplified analogues of such cAMPs. Up to now, only few monomers have been used in REP because of some limitations related to the solvents used (in which, for example, poly(sarcosine) was not soluble). ${ }^{29,30}$ Considering that both the hydrophobic and cationic characters are key structural features to design antimicrobial polymers,${ }^{31}$ we consequently prepared copolymers by mixing $N$-protected-lysine-like NNCA (Z- $N$-lys-NCA) and phenylalanine-like NNCA ( $N$-phe-NCA). Monomers were prepared through an optimized 3-step synthesis method (ESI). The LiHMDS-mediated REP of Z-Nlys-NCA was carried out in DMF at M/LiHMDS $=50$, alone (polymer 10 table 2 and ESI) or in copolymerization with $N$-phe-NCA, either one-pot (leading to statistical copolymers $\mathbf{1 1}$ to $\mathbf{1 5}$, table 2 ) or sequentially (leading to block copolymers $\mathbf{1 6}$ to $\mathbf{2 0}$, table 2). For all copolymers, acidic deprotection of the $\mathrm{Cbz}$ group ( $\mathrm{HBr} 2$ equiv. in TFA) afforded side-chain deprotection, allowing producing cationic macrocycles showing molar masses and chemical compositions, in agreement with the feed ratios (table S6 and figure S25).

The bioactivity of the copolymers was then assessed. Cyclic peptoids are promising scaffolds to design anti-infective agents, ${ }^{32,33}$ but antibacterial copolymers have never been developed based on this chemical design. We targeted a pathogenic Grampositive bacterium responsible for severe hospital acquired intestinal infections: Clostridioides difficile (C. difficile $)^{34,35}$ and copolymers 11-21 were evaluated in vitro against this pathogen (table 2 ). In this study, the bacteria were incubated for $24 \mathrm{~h}$ with increasing concentrations of cyclic polypeptoids, to determine the minimum inhibitory concentrations (MICs). In parallel, the in vitro cytotoxicity of the best polymers (15, 19-21) was tested on a human intestinal epithelial cell line (Caco-2), allowing to measure the $50 \%$ cytotoxic concentrations $\left(\mathrm{CC}_{50}\right)$ and calculate the selectivity indices $\left(\mathrm{SI}=\mathrm{CC}_{50} / \mathrm{MIC}\right)$. Optimizing the cationic content of copolymers was a key design to optimize the antibacterial activity and copolymer 15 showed a good profile, similar to the ones of LL37 (linear AMP, table 2) or antibiotic controls (table S7). Interestingly, this statistical copolymer $\mathbf{1 5}$ was significantly less toxic than copolymer diblock counterparts (19-20) or than the linear counterpart 21, (linearization of $\mathbf{1 5}$ by propylation, figure S26). We also observed that compound $\mathbf{1 5}$ preserved its activity after trypsin digestion, in marked contrast to LL37 (figure S28). This resistance to proteolysis and the ability to destabilize phospholipid membranes were further confirmed by in vitro assays involving liposomes (figure S29). Overall, this proof-of-concept demonstration shows that LiHMDS-mediated REP enables quick and simple access to libraries of copolymers to design antiinfective backbones.

Table 2. In vitro antibacterial activity against $C$. difficile and cytotoxicity over Caco-2 cell line (r: random copolymers and b: block copolymers).

\begin{tabular}{lcccc}
\hline $\begin{array}{c}\text { Polymer } \\
(r: \text { random \& } \\
b: \text { block })\end{array}$ & $\begin{array}{c}\text { Cationic } \\
\text { content } \\
(\%)^{\mathrm{a}}\end{array}$ & $\begin{array}{c}C \text {. diff. } \\
\mathrm{MIC} \\
(\mu \mathrm{g} / \mathrm{mL})\end{array}$ & $\begin{array}{c}\text { Caco-2 } \\
\mathrm{CC}_{50} \\
(\mu \mathrm{g} / \mathrm{mL})\end{array}$ & $\mathrm{SI}^{\mathrm{b}}$ \\
\hline $\mathrm{P}(N$-lys $) \mathbf{1 0}$ & 100 & $>100^{\mathrm{c}}$ & - & - \\
$\mathrm{P}(N$-lys- $r-N$-phe $) \mathbf{1 1}$ & $93(90)$ & $>100^{\mathrm{c}}$ & - & -
\end{tabular}


$\mathrm{P}(N$-lys- $r$ - $N$-phe $) 12$

$\mathrm{P}(N$-lys- $r-N$-phe $) 13$

$\mathrm{P}(N$-lys- $r-N$-phe $) 14$

$\mathrm{P}(N$-lys- $r-N$-phe $) \mathbf{1 5}$

15 post-trypsin

$\mathrm{P}(N$-lys- $b-N$-phe $) 16$

$\mathrm{P}(N$-lys- $b-N$-phe $) 17$

$\mathrm{P}(N$-lys- $b-N$-phe $) \mathbf{1 8}$

$\mathrm{P}(N$-lys- $b-N$-phe $) 19$

$\mathrm{P}(N$-lys- $b-N$-phe $) 20$

$\mathrm{P}(N$-lys- $r-N$-phe $) 21$

$\mathrm{LL}^{3}{ }^{\mathrm{d}}$

LL37 post-trypsin

Doxorubicin $^{\mathrm{e}}$

${ }^{\mathrm{a}}$ From ${ }^{1} \mathrm{H}$ NMR (in bracket, targeted) ${ }^{\mathrm{b}}$ Selectivity index $=\mathrm{CC}_{50} / \mathrm{MIC}$; ${ }^{\mathrm{c}}$ The $\mathrm{MIC}$ value was not reached at the highest tested concentration; ${ }^{\mathrm{d}}$ LL37 was used as AMP reference, ${ }^{\text {DD }}$ oxorubicin was used as a cytotoxic drug control.

In summary, the use of a LiHMDS-mediated REP of NNCA in DMF was a versatile and facile route to cyclic polypeptoids allowing fine-tuning over their chemical composition. This new methodology permits to design cationic macrocycles showing good activity against $C$. difficile, the main causative agent of nosocomial diarrheas of adults in developed countries. Overall, the use of LiHMDS is a simple way to design original protease-resistant backbones with proper bioactivity.

\section{AUTHOR INFORMATION}

\section{Corresponding Author}

*colin.bonduelle@enscbp.fr

*pierre.verhaeghe@univ-tlse3.fr

\section{Funding Sources}

PSA received support from CONACYT (scholarship holder No. 548662). This work was supported by the French national agency for research (ANR): grant $\mathrm{N}^{\circ}$ ANR-17-CE07-0039-01.

\section{ACKNOWLEDGMENT}

The authors acknowledge Sylvain Bourasseau for assistance with size-exclusion chromatography, AnneLaure Wirotious for NMR analysis and Joan Vignolle for helpful discussions. Christelle Absalon for MALDI TOF analysis. This work has benefited from the facilities and expertise of the CESAMO platform (Bordeaux University). This work was also granted access to the HPC resources of CALMIP supercomputing center under the allocation 2020 - P19021.

\section{ORCID numbers}

Pedro Salas-Ambrosio: 0000-0002-0922-4620
Antoine Tronnet: 0000-0002-7158-9305

Marc Since: 0000-0003-3984-5812

Sandra Bourgeade-Delmas: 0000-0001-9027-765X

Jean-Luc Stigliani: 0000-0002-9916-689X

Amélie Vax: 0000-0002-4766-7908

Sebastien Lecommandoux: 0000-0003-0465-8603

Bruno Dupuy: 0000-0001-7176-8453

Pierre Verhaeghe: 0000-0003-0238-2447

Colin Bonduelle: 0000-0002-7213-7861

\section{REFERENCES}

(1) Culkin, D. A.; Jeong, W.; Csihony, S.; Gomez, E. D.; Balsara, N. P.; Hedrick, J. L.; Waymouth, R. M. Zwitterionic Polymerization of Lactide to Cyclic Poly(Lactide) by Using N-Heterocyclic Carbene Organocatalysts. Angew. Chem, Int. Ed. 2007, 46 (15), 2627-2630.

(2) Honda, S.; Yamamoto, T.; Tezuka, Y. TopologyDirected Control on Thermal Stability: Micelles Formed from Linear and Cyclized Amphiphilic Block Copolymers. J. Am. Chem. Soc. 2010, 132 (30), 10251-10253.

(3) Honda, S.; Yamamoto, T.; Tezuka, Y. Tuneable Enhancement of the Salt and Thermal Stability of Polymeric Micelles by Cyclized Amphiphiles. Nat. Commun. 2013, 4.

Hoskins, J. N.; Grayson, S. M. Synthesis and Degradation Behavior of Cyclic Poly $(\varepsilon-$ Caprolactone). Macromolecules 2009, 42 (17), 6406-6413.

Borsali, R.; Benmouna, M. Static and Dynamic Scattering from Cyclic Diblock Copolymer Chains in Solution. Macromol. Symp. 1994, 79 (1), 153-166.

Borsali, R.; Lecommandoux, S.; Pecora, R.; Benoît, H. Scattering Properties of Rod-Coil and Once-Broken Rod Block Copolymers. Macromolecules 2001, 34 (12), 4229-4234.

(7) Haque, F. M.; Grayson, S. M. The Synthesis, Properties and Potential Applications of Cyclic Polymers. Nat. Chem. 2020, 12 (5), 433-444.

(8) Dove, A. P. Controlled Ring-Opening Polymerisation of Cyclic Esters: Polymer Blocks in Self-Assembled Nanostructures. Chem. Commun. 2008, 48, 6446.

(9) Laurent, B. A.; Grayson, S. M. Synthetic Approaches for the Preparation of Cyclic Polymers. Chem. Soc. Rev. 2009, 38 (8), 22022213.

(10) Kricheldorf, H. R. Cyclic Polymers: Synthetic Strategies and Physical Properties. J. Polym. Sci. Part A Polym. Chem. 2010, 48 (2), 251-284.

(11) Iatrou, H.; Hadjichristidis, N.; Meier, G.; Frielinghaus, H.; Monkenbusch, M. Synthesis and Characterization of Model Cyclic Block Copolymers of Styrene and Butadiene. 
Comparison of the Aggregation Phenomena in Selective Solvents with Linear Diblock and Triblock Analogues. Macromolecules 2002, 35 (14), 5426-5437.

(12) Schappacher, M.; Deffieux, A. $\alpha$-Acetal- $\omega$ Bis(Hydroxymethyl) Heterodifunctional Polystyrene: Synthesis, Characterization, and Investigation of Intramolecular End-to-End Ring Closure. Macromolecules 2001, 34 (17), 58275832.

(13) Schappacher, M.; Deffieux, A. Synthesis, Characterization, and Intramolecular End-to-End Ring Closure of $\alpha$-Isopropylidene-1,1Dihydroxymethyl- $\omega$-Diethylacetal PolystyreneBlock-Polyisoprene Block Copolymers. Macromol. Chem. Phys. 2002, 203 (17), 24632469.

(14) Jeong, W.; Hedrick, J. L.; Waymouth, R. M. Organic Spirocyclic Initiators for the RingExpansion Polymerization of $\beta$-Lactones. J. Am. Chem. Soc. 2007, 129 (27), 8414-8415.

(15) Bielawski, C. W.; Benitez, D.; Grubbs, R. H. An "Endless" Route to Cyclic Polymers. Science 2002, 297 (5589), 2041-2044.

(16) Piedra-Arroni, E.; Ladavière, C.; Amgoune, A.; Bourissou, D. Ring-Opening Polymerization with $\mathrm{Zn}(\mathrm{C} 6 \mathrm{~F} 5$ ) 2 -Based Lewis Pairs: Original and Efficient Approach to Cyclic Polyesters. J. Am. Chem. Soc. 2013, 135 (36), 13306-13309.

Zhang, Y.; Liu, R.; Jin, H.; Song, W.; Augustine, R.; Kim, I. Straightforward Access to Linear and Cyclic Polypeptides. Commun. Chem. 2018, 1 (1), 1-7.

(18) Guo, L.; Zhang, D. Cyclic Poly( $\alpha$-Peptoid)s and Their Block Copolymers from N-Heterocyclic Carbene-Mediated Ring-Opening Polymerizations of N-Substituted N Carboxylanhydrides. J. Am. Chem. Soc. 2009, 131 (50), 18072-18074.

(19) Chan, B. A.; Xuan, S.; Li, A.; Simpson, J. M.; Sternhagen, G. L.; Yu, T.; Darvish, O. A.; Jiang, N.; Zhang, D. Polypeptoid Polymers: Synthesis, Characterization, and Properties. Biopolymers 2017, 109 (1), 1-25.

(20) Li, A.; Lu, L.; Li, X.; He, L. L.; Do, C.; Garno, J. C.; Zhang, D. Amidine-Mediated Zwitterionic Ring-Opening Polymerization of $\mathrm{N}$-Alkyl $\mathrm{N}$ Carboxyanhydride: Mechanism, Kinetics, and Architecture Elucidation. Macromolecules 2016, 49 (4), 1163-1171.

(21) Guo, L.; Lahasky, S. H.; Ghale, K.; Zhang, D. N -Heterocyclic Carbene-Mediated Zwitterionic Polymerization of $N$-Substituted $N$ Carboxyanhydrides toward $\operatorname{Poly}(\alpha$-Peptoid)s: Kinetic, Mechanism, and Architectural Control. J. Am. Chem. Soc. 2012, 134 (22), 9163-9171.

(22) Wu, Y.; Zhang, D.; Ma, P.; Zhou, R.; Hua, L.;
Liu, R. Lithium Hexamethyldisilazide Initiated Superfast Ring Opening Polymerization of Alpha-Amino Acid N-Carboxyanhydrides. Nat. Commun. 2018, 9 (1), 5297.

Boyd, G. V.; Wright, P. H.; Boyd, G. V.; Wright, P. H. Unstable Mesoionic Oxazolium-5-Oxides. J. Chem. Scociety Perkin I 1972, No. 914, 914918.

(24) Gingrich, H. L.; Baum, J. S. Mesoionic Oxazoles. Chemistry of Heterocyclic Compounds. January 2, 1986, pp 731-961.

(25) Alberty, K. A.; Hogen-Esch, T. E.; Carlotti, S. Synthesis and Characterization of Macrocyclic Vinyl-Aromatic Polymers. Macromol. Chem. Phys. 2005, 206 (10), 1035-1042.

(26) Lutz, P.; McKenna, G.; Rempp, P.; Strazielle, C. Solution Properties of Ring-shaped Polystyrenes. Die Makromol. Chemie, Rapid Commun. 1986, 7 (9), 599-605.

(27) Sprott, G. D. Structures of Archaebacterial Membrane Lipids. J. Bioenerg. Biomembr. 1992, 24 (6), 555-566.

(28) Hemu, X.; Qiu, Y.; Nguyen, G. K. T.; Tam, J. P. Total Synthesis of Circular Bacteriocins by Butelase 1. J. Am. Chem. Soc. 2016, 138 (22), 6968-6971.

(29) Lee, C.-U.; Li, A.; Ghale, K.; Zhang, D. Crystallization and Melting Behaviors of Cyclic and Linear Polypeptoids with Alkyl Side Chains. Macromolecules 2013, 46 (20), 8213-8223.

(30) Lahasky, S. H.; Serem, W. K.; Guo, L.; Garno, J. C.; Zhang, D. Synthesis and Characterization of Cyclic Brush-Like Polymers by $N$-Heterocyclic Carbene-Mediated Zwitterionic Polymerization of $N$-Propargyl $N$-Carboxyanhydride and the Grafting-to Approach. Macromolecules 2011, 44, 9063-9074.

(31) Salas-Ambrosio, P.; Tronnet, A.; Verhaeghe, P.; Bonduelle, C. Synthetic Polypeptide Polymers as Simplified Analogues of Antimicrobial Peptides. Biomacromolecules 2021, 22 (1), 57-75.

Benson, M. A.; Shin, S. B. Y.; Kirshenbaum, K.; Huang, M. L.; Torres, V. J. A Comparison of Linear and Cyclic Peptoid Oligomers as Potent Antimicrobial Agents. ChemMedChem 2012, 7 (1), 114-122.

(33) Miller, S. M.; Simon, R. J.; Ng, S.; Zuckermann, R. N.; Kerr, J. M. Comparison of the Proteolytic Susceptibilities of Homologous L-Amino Acid, $D$-Amino Acid and $N$-Substituted Glycine Peptide and Peptoid Oligomers. Drug Dev. Res. 1995, 32, 20-32.

(34) Liu, R.; Suárez, J. M.; Weisblum, B.; Gellman, S. H.; McBride, S. M. Synthetic Polymers Active against Clostridium Difficile Vegetative Cell Growth and Spore Outgrowth. J. Am. Chem. Soc. 2014, 136 (41), 14498-14504. 
Centers for Disease Control and Prevention (CDC). Antibiotic Resistance Threats in the United States. Antibiotic resistance threats in the United https://www.cdc.gov/drugresistance/biggest-

threats.html (accessed May 15, 2020). 